\title{
Geo-neutrino, Earth heat flux, Earth electricity
}

\author{
L. B. Bezrukov ${ }^{1, *}$, A. S. Kurlovich ${ }^{1}$, B. K. Lubsandorzhiev ${ }^{1}$, V. V. Sinev ${ }^{1}$, V. P. Zavarzina ${ }^{1}$, \\ and V.P. Morgalyuk ${ }^{2}$, \\ ${ }^{1}$ Institute for Nuclear Researches of Russian Academy of Sciences, Prospekt 60-letia Oktyabrya 7a, \\ Moscow, 115409, Russia \\ ${ }^{2}$ A. N. Nesmeyanov Institute of Organoelement Compounds of Russian Academy of Sciences, Vavilova \\ 10, Moscow, 115409, Russia
}

\begin{abstract}
We discussed the idea of big value of potassium abundance in the Earth. We showed that Borexino single event spectrum permit the potassium abundance up to $2 \%$ instead of the CNO neutrino flux contribution. Works [5], [6] introduce the idea that fast $\alpha$ particles are appeared in nuclear processes in solar core plasma. The reactions of these $\alpha$ particles with CNO nuclei can suppress the CNO neutrino flux. We demonstrated the connection between the existence of Earth's electric field and the big value of ${ }^{40} \mathrm{~K}$ geo-neutrino flux because the both phenomena are the sequences from Hydride Earth model.
\end{abstract}

\section{Introduction}

In the previous 19th International Seminar on High Energy Physics, Quarks-2016, we discussed the problem of geo-neutrino [3]. In this work we demonstrated that the experimental data on geo-neutrinos allow to admit that masses of $\mathrm{U}$, Th and $\mathrm{K}$ in the Earth can be up to $m_{U}=1.7 \cdot 10^{17} \mathrm{~kg}, m_{T h}=6.7 \cdot 10^{17} \mathrm{kgand}_{\mathrm{K}} / m_{\text {Earth }} \sim 2 \%$. These values correspond to intrinsic Earth heat flux in $\sim 300 \mathrm{TW}$. The most part of this flux goes up in rift zones in the form of the heated gases. Argo Project results and the measurements of the Moon intrinsic heat flux support the existence of such a big flux. The Hydride Earth (HE) model [1] predicts so large of $\mathrm{U}, \mathrm{Th}, \mathrm{K}$ abundances.

In the work [4] we introduce the new mechanism of heat transfer in the Earth crust: the energy transfer by hot gases created in the Earth crust at great depth. The temperature profiles obtained in the Kola super-deep borehole support this idea.

Here we will continue the discussion about the prediction of so large of $\mathrm{K}$ abundance in the Earth.

\section{Potassium abundance problem}

BSE model does not give the large value of potassium abundance in total Earth mass. It is considered that all potassium is concentrated in the crust. Potassium abundance in the crust is about $2 \%$ in weight. But the absence of potassium inside of Earth is based on the fact that meteorites have low abundance of potassium. In reality we have no information about potassium abundance inside the Earth below the crust. If its abundance in the Earth is about

*e-mail: bezrukov@inr.ac.ru 
the same as in the crust the thermal flux could be much more larger than predicted BSE model. The only way to obtain information on the potassium inside the Earth is to measure antineutrino and possibly neutrino fluxes from ${ }^{40} \mathrm{~K}$ which content in natural potassium is $1.17 \times 10^{-4}$.

Calculated heat flux from ${ }^{40} \mathrm{~K}$ is ten times larger than one from ${ }^{238} \mathrm{U}$ and ${ }^{232} \mathrm{Th}$ in sum in case of its abundance on the level of $2 \%$ all over the Earth.

Thermal flux from ${ }^{40} \mathrm{~K}$ can be nondirectly checked by registering antineutrino and neutrino fluxes produced by ${ }^{40} \mathrm{~K}$. In [2] the spectrum of recoiled electrons from ${ }^{40} \mathrm{~K}$ was compared with spectra from other neutrino sources in Borexino detector.

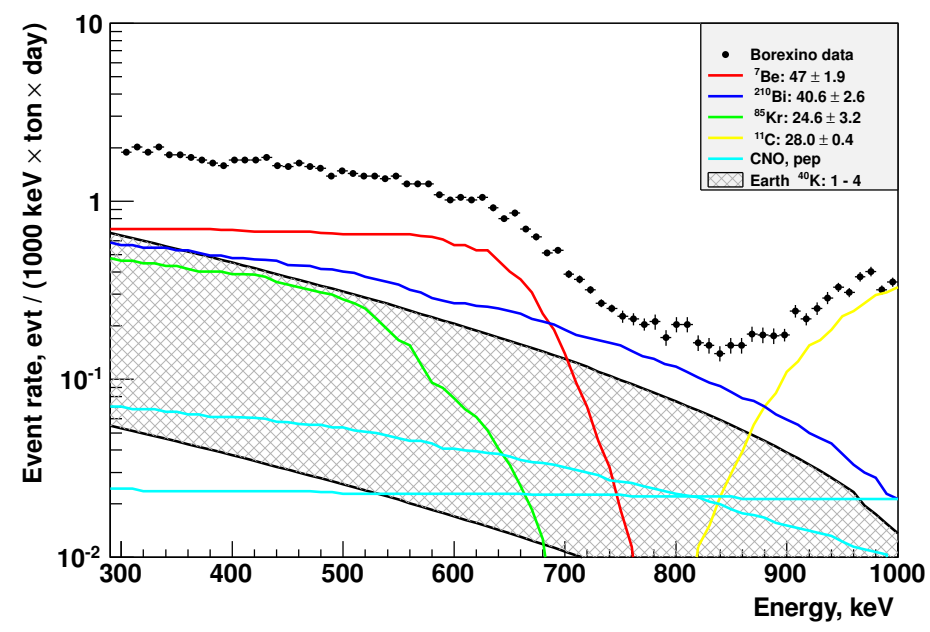

Figure 1. An analytic fit over $290-1270 \mathrm{keV}$ energy region to experimental spectrum obtained in [7]. The numbers are the count rate per day per $100 \mathrm{t}$ for different sources of count rate. The black lines are the result of our calculations [2], [4] of the possible contribution of ${ }^{40} \mathrm{~K}$ geo-antineutrino in count rate of single events of Borexino detector. The upper black line corresponds to $4 \%$ of $\mathrm{K}$ in the Earth mass, the bottom black line corresponds to $1 \%$ of $\mathrm{K}$ in the Earth mass.

Fig. 1 shows an analytic fit over $290-1270 \mathrm{keV}$ energy region to experimental spectrum obtained in [7]. The numbers are the count rate per day per $100 \mathrm{t}$ for different sources of count rate. The black lines are the result of our calculations [2], [4] of the possible contribution of ${ }^{40} \mathrm{~K}$ geo-antineutrino in count rate of single events of Borexino detector. The upper black line corresponds to $4 \%$ of $\mathrm{K}$ in the Earth mass, the bottom black line corresponds to $1 \%$ of $\mathrm{K}$ in the Earth mass. We can see from fig. 1 that in the case of $2 \%$ of $\mathrm{K}$ in the Earth mass the contribution of ${ }^{40} \mathrm{~K}$ geo-antineutrino in count rate of single events of Borexino detector is the same as the contribution from CNO solar neutrino. If we believe that Standard Solar Model is true we can say, that the existence of $2 \%$ of $\mathrm{K}$ in the Earth mass is wrong idea. On the contrary, if we believe that $2 \%$ of $\mathrm{K}$ in the Earth mass is correct idea the CNO neutrino flux must be lower than the Standard Solar Model CNO neutrino flux. Refer here to results of works [5], [6] in which the authors introduce the idea about fast $\alpha$ particles appearing in nuclear processes in solar core plasma. The reactions of these $\alpha$ particles with CNO nuclei can suppress the CNO neutrino flux. If this result is correct the Borexino detector really see the ${ }^{40} \mathrm{~K}$ geo-antineutrino now. 


\section{Connection the geo-neutrino fluxes and Earth electricity, Hydridc Earth model}

The idea of big potassium abundance in the Earth follows from Hydride Earth (HE) model (or Hydrogen rich primordial Earth) [1]. To check the truth of HE model and as a sequence the truth of the statement about the big potassium abundance we found the new phenomenon predicted by HE model. We experimentally observed this phenomenon.

Based on the HE model, we propose a Hydride Earth Electricity (HEE) model [8]. HEE predicts that the negative electrode of the Earth's capacitor is located under the Earth's crust and the Earth's fluids carry a positive charge. We have observed an excess of positive charge in the Earth's crust down to kilometer depths. The model explains the unitary variation of the fair-weather atmospheric electric field strength, the change in atmospheric electric field strength and the precipitation of high-energy electrons during the earthquakes.

We demonstrated the connection between the existence of Earth's electric field and the big value of ${ }^{40} \mathrm{~K}$ geo-antineutrino flux. As a sequence of this fact it is follows from Borexino single event spectrum that the CNO neutrino flux must be lower than the Standard Solar Model CNO neutrino flux.

\section{Conclusion}

1. The Hydride Earth model predicts the huge amount of $\mathrm{K}$ in the Earth. ${ }^{40} \mathrm{~K}$ geoantineutrino must contribute to Borexino single events at the level of $2-3$ events per day. The suppression of CNO neutrino flux follows from Borexino results in this case. The reactions with fast $\alpha$ particles in the Sun can be the reason of such suppression.

2. We developed the Hydride Earth Electricity (HEE) model. We proved the HEE experimentally. We observed the unusual predicted phenomenon: The Earth crust (continents and ocean) saturates by positive ions (protons).

3. We demonstrated the connection between the existence of Earth's electric field and the big value of ${ }^{40} \mathrm{~K}$ geo-neutrino flux because the both phenomena are the sequences from Hydride Earth model.

\section{Acknowledgements}

We are grateful to Igor Tkachev and Viktor Voronchev for useful discussions.

\section{References}

[1] V. N. Larin (ed. C. Warren Hunt), Hydridic Earth: the New Geology of Our Primordially Hydrogen-Rich Planet (Polar Publishing, Calgary, Alberta, Canada, 1993) 247.

[2] V. V. Sinev, L. B. Bezrukov, E. A. Litvinovich, I. M. Machulin, M. D. Skorokhvatov, S. V. Sukhotin, Phys. Part. and Nucl. 46, 186 (2015)

[3] L. B. Bezrukov, A. S. Kurlovich, B. K. Lubsandorzhiev, V. V. Sinev, V. P. Zavarzina and V. P. Morgalyuk, EPJ Web of Conferences 125, 02004 (2016)

[4] L. B. Bezrukov, A. S. Kurlovich, B. K. Lubsandorzhiev, A. K. Mezhokh, V. P. Morgalyuk, V. V. Sinev, V.P. Zavarzina, Phys. Part. Nucl. 49, 674 (2018); Original Russian Text published in Fizika Elementarnykh Chastits i Atomnogo Yadra, 49, 1191 (2018)

[5] V. T. Voronchev, Y. Nakao, and Y. Watanabe, Phys. Rev. C 96, 055803 (2017) 
[6] V. T. Voronchev, Y. Nakao, and Y. Watanabe, J. of Physics G: Nuclear and Particle Physics 44, 045202 (2017)

[7] M. Derbin and V. Muratova on behalf of Borexino Collaboration, arXiv:1605.06795v1 [hep-ex]

[8] L.B. Bezrukov, V.P. Zavarzina, A.S. Kurlovich, B.K. Lubsandorzhiev, A.K. Mezhokh, V.P. Morgaluk, V.V. Sinev, Doklady Physics 63, No. 5, 177 (2018); Original Russian Text published in Doklady Akademii Nauk 480, No. 2, 155 (2018) 